\title{
A Real Pilot-Platform Implementation for Blockchain-Based Peer-to-Peer Energy Trading
}

\author{
Islam El-Sayed \\ Electrical Eng. Dept. \\ University of Oviedo \\ Gijón, Spain
}

\author{
Komal Khan \\ Electrical Eng. Dept. \\ University of Oviedo \\ Gijón, Spain
}

\author{
Xavier Dominguez \\ Facultad de Ingeniería \\ Univ. Técnica del Norte \\ Ibarra, Ecuador
}

\author{
Pablo Arboleya \\ Electrical Eng. Dept. \\ University of Oviedo \\ Gijón, Spain
}

\begin{abstract}
The ever growing energy demand due to population growth, higher penetration of electric vehicles and smart appliances, as well as superior living standards, is a demanding incentive to the better utilization of conventional and renewable energy systems. Moreover, to facilitate the emerging requirements of prosumers to participate in the electricity market and monetise their efforts towards distributed energy deployment, traditional centralised energy trading architectures are no longer viable. In this context, blockchain-based ledger technology emerges as the most feasible solution which offers a peer to peer (P2P) energy trading platform providing a unique distributed local energy market model for beneficial energy exchanges among participants. This will represent a significant evolution for future smart grids. In this regard, this work provides a ground understanding as well as all the necessary technical details and procedures required to implement a pilot-platform P2P energy trading system based on blockchain technology. All the source codes have been uploaded and socialized. This may support academics and entrepreneurs at the initial development stage of these kind of initiatives.
\end{abstract}

Index Terms - blockchain, energy trading, peer-to-peer, transactive energy, ethereum.

\section{INTRODUCTION}

In the last years, a sustained increasing use of renewable energy sources (RES) has been witnessed worldwide. Moreover, by 2022 around 30 percent of the overall electricity production will come from RES [1]. Nonetheless, the electricity demand is expected to increase by 20 percent in the next decade as a consequence of population growth, higher penetration of electric vehicles (EVs) and smart appliances, as well as superior living standards [2]. In the pursue to face this challenging scenario and properly meet the renewable energy generation with the demand, the microgrid concept was proposed as a convenient alternative. Nevertheless, microgrids present some difficulties on its coordination and control when they are connected to the conventional grid by means of network operators and utility companies which in most cases impose high logistics and costs for the electricity use. To overcome this issue, the latest advancements in digital communication and measurement systems added to the IoT technology have contributed to the development of smart grid infrastructures that permit a safe and reliable energy exchange between energy players (producers, consumers and

Corresponding author email: arboleyapablo@uniovi.es

This work was partially supported by the Spanish Ministry of Economy and Competitivity under grant MINECO-17-ENE2016-77919-R (CONCIALIATOR Energy conversion technologies in resilient hybrid AC/DC distribution networks), by the Government of the Principality of Asturias - Foundation for Scientific and Technical Research (FICYT) under grants FC-GRUPIN-IDI/2018/000241 (Laboratory for Enhanced Microgrid Unbalance Research (LEMUR)) and BP19-069 ("Severo Ochoa" Program of Pre-Doctoral Grants) and by Phoenix Contact Foundation. prosumers) [3]. In this context, the ongoing challenge consists on decentralizing the energy production and consumption. To do so, energy blockchain has been exhibited as the required disrupting technology towards a new paradigm change in the power industry. Indeed, Blockchain 2.0 will permit the democratisation of power systems by means of the peer-to-peer (P2P) energy trading and the smart contract technology [4]. In turn this will promote local electricity trade, the reduction of electricity transmission losses, optimization of power flow, grid stability improvements, demand-side management and cost-effective employment of distributed energy [5]. Furthermore, P2P trade represents a win-win situation for prosumers but also for typical consumers as energy prices could be agreed with lower values compared with the ones defined by conventional electrical markets [3].

In this context, several systematic efforts have been exhibited regarding the use of blockchain, smart contracts and $\mathrm{P} 2 \mathrm{P}$ in the power energy sector in the last years. The various applications, advantages and use cases when using blockchain are exhibited in [6] and [7]. References [8], [9] and [10] deal with adding privacy and security in energy market transactions by means of providing smart contract algorithms, decentralized knowledge graph construction and public-key cryptosystems respectively. Other security aspects for secure energy delivery and a credit-based payment schemes are analyzed in [11] and [12]. In [13], the benefits of using blockchain when procuring voltage regulation with reactive power control are mentioned. A further ancillary services discussion is held in [14] where relevant distributed ledger platforms for energy transaction in microgrids are also discussed. A proposal to perform continuous double auction market to match the distributed generation (DG) offer and demand is detailed in [15]. There, blockchain is proposed to cooperate with financial institutions by means of a multi signature system. Workflows and algorithms for developing agent coalition and electricity negotiation mechanisms are presented in [16]. In [17], thousands of smart contracts are analyzed. The ones having greater transactions are discussed. An adaptive blockchain-based electric vehicle participation (AdBEV) scheme is proposed in [18]. A framework based on a blockchain network able to carry on simulation of market clearing operations with a payment process is exhibited in [19]. Blockchain application's frameworks for crowdsourced energy systems and smart grid data security have been reviewed in [20] and [6] correspondingly.

The aforementioned references provide different frameworks and concepts to guide the planning for blockchain-based projects at the power energy sector. However, they lack on providing detailed technical explanations to implement a real 
pilot platform blockchain-based system able to perform P2P energy trading, which is in turn the aim of this work. Specific technical guidelines to deploy P2P energy projects would be of high significance for academics and entrepreneurs to fasten the development process at the initial stage. To achieve this goal while providing a comprehensive understanding, this work is organized as follows. Section II specifies the key foundations to unfold a blockchain-based P2P energy project. Physical and Virtual network requirements with the related hardware and software tools are detailed in Section III. A detailed implementation methodology is exhibited in Section IV. Finally, some conclusions are inferred in Section V.

\section{BLOCKCHAIN AS A KEY-ENABLER FOR PEER-TO-PEER ENERGY PLATFORMS}

A. Transactive Energy Using Blockchain. Core Technologies. The advent of smartgrids and the participation of more prosumers in the electricity market have increased the requirements for enabling energy transactions among all players [21] and thus promoting a decentralised vision for the power grid by means of Transactive Energy (TE) platforms.

Indeed, this technology could turn conventional power grids into modernised systems which expedite the collaboration of the different participants in the network. This qualitative improvement could be attained in coming years thanks to the rapid and constant evolution of the blockchain technology. Hereof, three blockchain generations have been traced [21]. In the first one, bitcoin was introduced. Then, in the second generation, the automated smart contract technology was presented by Ethereum to process and record any logical operation in a reliable and secured ledger.

In these last years, Blockchain 3.0 has been exposed as a mature technology which has been already employed in some P2P TE pilot projects [22]-[24]. The main technological foundations that support this disrupting energy trading innovation are:

1) IoT and Energy Digitalisation: Around 20 billion smart devices will be worldwide connected to the internet by 2020 [25]. To face this challenge and deploy advanced power metering infrastructure, an increasing number of smart meters and information-communication technologies (ICTs) are being installed [25]. Smart meters integrated with blockchain can ease to record and track the data on temper-proof ledgers at suitable time intervals. Moreover, some companies are offering smart devices having ultra high resolution sampling and also including controllability via mobile applications [26].

2) Cloud and Edge Computing: A reliable transactive control in local energy markets is being affordably achieved by virtue of cloud and edge computing as they permit autonomous contract depletion for network edge users and avoid the need for trusted third party platforms [27].

3) Big Data and Artificial Intelligence: By means of artificial intelligence $(\mathrm{AI})$, optimized automated decision-making can be achieved for energy players according to their needs, energetic patterns, weather forecast and storage conditions. Moreover, due to the increasing volumes of information, advanced computing, data mining and machine learning are required for handling big data [28].

\section{B. Initiatives, Platforms and Challenges}

In the last years, several startups and pilot projects have been developed under the P2P energy trading umbrella taking advantage of the blockchain decentralised architecture. The most renowned initiatives are Power Ledger, LO3,
Grid+ and Verv. However, there are tens of companies, foundations and consortiums related to blockchain-based energy projects as references [29] and [30] exhibit. On the other hand, some collaborative platforms have been established by some organizations to extend blockchain applications. Among these, the most popular are the Ethereum-based Energy Web Chain software and the Linux-based Hyper Ledger framework. Reference [30] provides further details on blockchain platforms employed in the energy sector.

Despite the fact that blockchain arises as a revolutionary application for P2P energy trading, it also presents some significant challenges. Most of them are related with security and privacy concerns as public blockchains are accessible for all the parties. Hence, novel solutions are needed to preserve anonymity and privacy so that energy usage data can not be traced by other individual users. In this regard, permissioned blockchain is emerging as a potential solution. Another relevant concern is the storage and processing of ever-increasing data that could not be handled with the current infrastructure capabilities. Last but not least, the role of transmission and distribution system operators (TSOs/DSOs) should be highlighted as key enablers of this revolution since they hold the physical infrastructure and operation of the system. They should support $\mathrm{P} 2 \mathrm{P}$ trading platforms to permit a decentralised access to the power grid given the physical constraints, but they are not required to centrally manage the energy transactions [30]. Therefore, proper coordination between TSOs/DSOs and the other energy players is crucial to permit a reliable and rapid incorporation of this technology. Once these logistic aspects are overcome, the role of AI will be again highly relevant to suitably forecast the energy supply and demand so that early adjustments in the system can take place even before the trading starts.

Since blockchain-based P2P energy trading is nowadays not mature enough to be adapted by the mainstream as it is still in early development stages, sustained research and contributions are needed to take progressive benefits of its fullest potential at a social, commercial and energetic level. In this respect, next sections detail an entire procedure to implement a blockchain-based P2P energy trading mainly intended for regulated markets but still flexible enough to be adapted in a non-regulated context.

\section{Pilot Platform Implementation}

\section{A. Physical and Virtual Networks}

A P2P transactive energy scheme is comprised of two systems, the physical and the virtual energy networks. The former is responsible for the physical transfer of energy between peers. This could be achieved by a distributed grid network managed by an independent system operator (ISO) or also by a separate microgrid tied to a conventional grid. On the other hand, the virtual network provides the blockchain-based architecture for energy trading platform capable to handle all kinds of data transfer related to electricity generation/consumption and buy/sell offers. The bids have to be matched and accepted for the payments to then take place. Payments are made by consumers to prosumers in order to inject their renewable energy into the grid.

The present work intends to impart technical implementation details of a simple yet practical demonstration on how the energy trade occurs between the peers. Moreover, all the source codes later explained have been uploaded to the IEEEDataPort repository and are accessible online at [31]. 


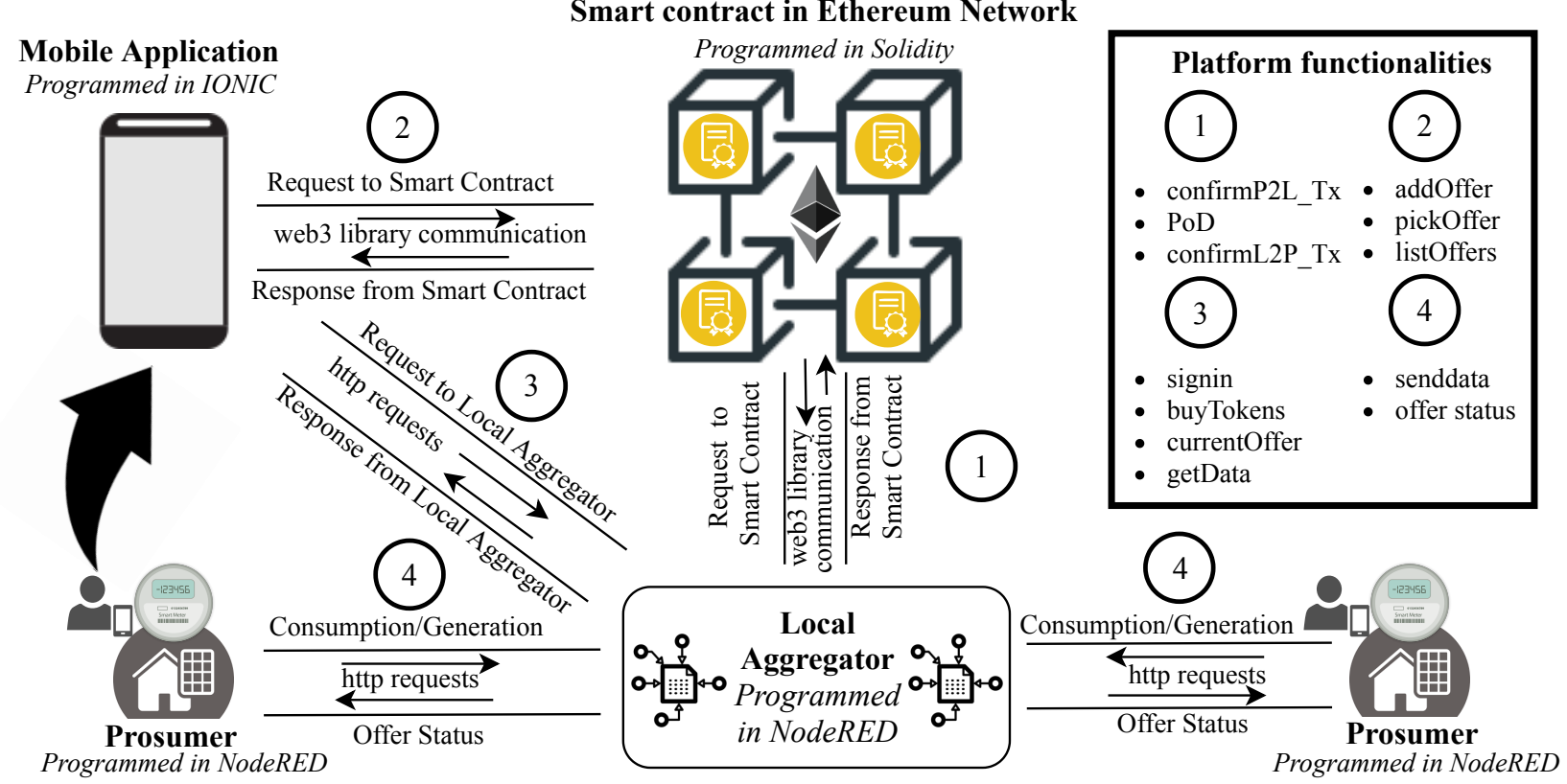

Fig. 1: Design Model of Blockchain-Based P2P Energy Trading using IoT Devices

To begin explaining the implementation, lets define the roles of the participants first:

1) Peers: They can be seen as the energy players who are selling or buying their surplus renewable energy . In turn they can be classified as:

- Consumer: A participant who merely consumes electricity.

- Prosumer: A participant owning a renewable energy system and thus producing and consuming electricity.

2) Local Aggregator: Wide P2P energy trading networks are usually divided into some communities where each of those possess its own local aggregator which acts as a broker and allows the peers within the community to trade electricity. Indeed, local aggregators buy tokens from public exchange and sell tokens locally to the participants on request.

\section{B. Case Scenario}

Considering the aforesaid, a simple scenario has been built under a regulated market scheme considering Peer A and Peer $\mathrm{B}$ as registered participants in our energy trade platform. To provide the participants an easy accessibility to perform the trading, a mobile application has been developed (the code of this app is also shared in the repository). The procedure is as follows:

- Peer A desires to sell his surplus energy. By means of our energy-trade platform he will make an offer through the mobile application.

- Peer B is interested in the offer and buys the energy via the mobile application.

- The local aggregator requests Peer $\mathrm{A}$ to begin exporting the energy while Peer B begins to consume energy.

- The local aggregator checks for the proof of delivery (PoD) making use of smart meter data on both ends.

- After PoD is confirmed, the local aggregator pays Peer A for the exported energy deducting the tokens available in the account of Peer B.
TABLE I: HARDWARE AND SOFTWARE TOOLS

\begin{tabular}{|c|}
\hline$\overline{\mathrm{H}}$ \\
\hline $\begin{array}{l}\text { Raspberry Pi } 3 \text { Model B+ is used as smart meter installed at the user's dwelling. } \\
\text { Initially, Arduino microcontroller was considered due to its compact size and tailored } \\
\text { development features compared to Raspberry Pi. However, the latter exhibited better } \\
\text { functionalities when dealing with Web3 library which is needed for communicating } \\
\text { with the smart contract. }\end{array}$ \\
\hline $\begin{array}{l}\text { Leds and Sense HAT 8x8 RGB LED matrix (add-on board of Raspberry Pi) are } \\
\text { used for indicating trading session and the energy transaction status. }\end{array}$ \\
\hline $\begin{array}{l}\text { Rotary potentiometers are manually handled to emulate the user's energy } \\
\text { consumption/production. }\end{array}$ \\
\hline $\begin{array}{l}\text { MCP3008 chip performs the analog to digital conversion of the potentiometer's } \\
\text { signals to be sent to the smart meter. }\end{array}$ \\
\hline $\begin{array}{l}\text { Local aggregator services are programmed in a server that stores the user's } \\
\text { smart meter data. Additionally, the service encompass payment agreements, token } \\
\text { exchanges and PoD operations. }\end{array}$ \\
\hline SOFTWARE \\
\hline $\begin{array}{l}\text { Energy Web Foundation (EWF) - Tobalaba Test Network is employed to build and } \\
\text { validate the smart contract. Moreover, EWF provisions the front-end Energy-Web } \\
\text { UI user interface for creating accounts, define smart contracts and test these } \\
\text { functionalities in the Ethereum Tobalaba network. }\end{array}$ \\
\hline $\begin{array}{l}\text { SQLite engine was used to create the smart meters and users database but also for } \\
\text { performing data queries (insert, update, select) to the database. }\end{array}$ \\
\hline $\begin{array}{l}\text { Node-RED development interface permitted a flow-based visual programming for } \\
\text { implementing the platform services such as local aggregator functionalities, smart } \\
\text { meter - data server communication and smart contract interplay. Furthermore, } \\
\text { Node-RED acts as back-end for the Ionic application. }\end{array}$ \\
\hline $\begin{array}{l}\text { Ionic is an open-source web application development environment. It was used to } \\
\text { build the mobile application using Angular framework. }\end{array}$ \\
\hline $\begin{array}{l}\text { Web3 JavaScript API allows to interact with the Ethereum blockchain network. } \\
\text { This library permits to retrieve user accounts, interact with smart contracts and } \\
\text { send/receive transactions among other features. }\end{array}$ \\
\hline
\end{tabular}

\section{Hardware and Software}

During the state-of-the-art review, a variety of tools were explored and considered. Ultimately, the alternatives exposed in Table I were adopted.

\section{Proposed Methodology}

A. Ethereum-based smart contract deployment

First of all, the Ethereum-based smart contract structure has to be deployed. This was achieved by means of the Energy Web Foundation (EWF) ecosystem. Indeed, EWF is the largest energy blockchain framework worldwide intended for regulatory energy sectors as well as for specific business 

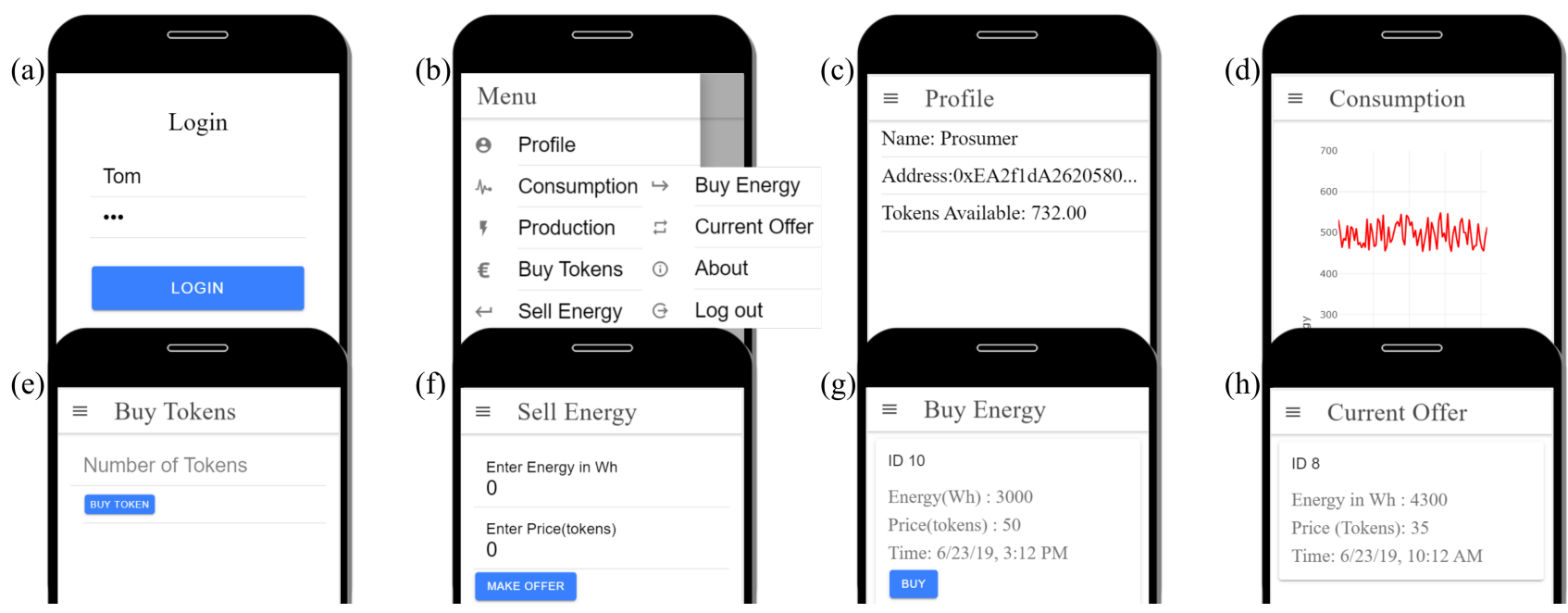

Fig. 2: Energy Trade Mobile Application Panels

TABLE II: ENERGY TRADE OFFER.

\begin{tabular}{|l||l|l|}
\hline \multicolumn{3}{|c|}{ Structure of an Offer } \\
\hline Size & Arguments & Details \\
\hline 4 bytes & ID & offer ID \\
20 bytes & seller & seller address \\
4 bytes & energy & amount of electricity for sale (Wh) \\
4 bytes & price & price of electricity for sale (tokens) \\
4 bytes & timeOffered & time when offer is added \\
\hline
\end{tabular}

and market needs [32]. To begin the implementation, from the EWF platform, Energy Web Client UI was downloaded and installed. This referred to a user interface which provides a desktop environment for connecting the peers in the blockchain network, creating accounts, sending transactions and interacting with smart contracts.

\section{B. Accounts Creation}

For the registered users and the local aggregator, Parity Ethereum wallet accounts were created using Energy Web UI. This environment redirects to Energy Web Tobalaba to issue fake tokens to developers for testing and deployment purposes regarding smart contracts.

\section{Smart Contract Creation}

Energy Web UI provides functionalities to develop smart contract. To implement the proposed scenario earlier described, the following functions of the smart contract were programmed using Solidity:

- addOffer: This function permits peers (prosumers) to create their offers by defining some details such as the intended amount of energy to be sold and its price. The structure of an offer is exhibited in Table II.

- pickOffer: If a peer (consumer) is interested in any offer, this function allows to confirm his choice and holds the offer in the system.

- confirmP2L_Tx: Now the status of the transaction (requested by the consumer to the local aggregator) is checked. Once the consumer payment is done, the confirmation is registered.

- POD: The local aggregator calls this function to confirm the energy delivery by the prosumer, provoking the subsequent process.

- confirmL2P_Tx: This function confirms the payment made by the local aggregator to the prosumer to successfully finish the P2P energy trade.
TABLE III: Mobile PANEL Views

\begin{tabular}{|l|l|}
\hline Panel & \multicolumn{1}{|c|}{ Explanation } \\
\hline \hline (a) & $\begin{array}{l}\text { It contains the login access. As part of the back-end, the mobile application } \\
\text { makes a signin http request to the local aggregator server. Once the } \\
\text { credentials are validated, the user us redirected correspondingly to the } \\
\text { menu. }\end{array}$ \\
\hline (b) & It displays the general menu of all services provided. \\
\hline (C) & $\begin{array}{l}\text { It exhibits the profile page, where the personal user data (name, address, } \\
\text { available tokens) is displayed so that a balance of the account can be } \\
\text { inferred. }\end{array}$ \\
\hline (d) & $\begin{array}{l}\text { It provides the user with real time monitoring regarding the energy } \\
\text { consumption/production. The mobile application makes get data http } \\
\text { request to the local aggregator server to have in turn the corresponding } \\
\text { smart meter data. }\end{array}$ \\
\hline (C) & $\begin{array}{l}\text { It permits the user to buy tokens from the local aggregator. } \\
\text { In this panel, the user is able to make an energy offer invoking a specific } \\
\text { function (Add offer) of the smart contract so that details such as } \\
\text { transaction ID, energy, price, time and user data are passed as arguments. }\end{array}$ \\
\hline (f) & $\begin{array}{l}\text { It shows the user a list of the different available offers with their details } \\
\text { (as in the previous panel). The back-end demands the smart contract list } \\
\text { offer function that in contrast return all the accessible energy offers. } \\
\text { Once the user selects an offer, the Pick of fer function is recalled so } \\
\text { that the user details are given as arguments. Then the offer is deleted from } \\
\text { the overall list and energy price is transferred from the user account to the } \\
\text { local aggregator account }\end{array}$ \\
\hline It displays the selected energy offer being now in the transaction process. \\
\hline (h)
\end{tabular}

\section{Design Model}

The proposed model was intended to create a practical demo of a local energy trading community under a regulated market scheme. To do so, four main components can be distinguished: (i) blockchain-based smart contract, (ii) the local aggregator, (iii) smart meters and (iv) the mobile application service. The correlation between these components is sketched in Figure 1.

\section{E. Advanced Metering}

As earlier mentioned, smart meters are modelled with Raspberry Pi's (programmed employing Node-Red visual programming) and the user's energy generation/consumption are emulated with potentiometers. Moreover, the user's smart meters are registered in the platform with a unique ID. They send their measured energy information to the local aggregator server once per second.

\section{F. Mobile Application}

The platform front-end interface is provided by the mobile application and it consists of different panel views for the different services as Table III and Figure 2 expose. 


\section{G. Local Aggregator Server}

Using Node-Red, the local aggregator services were programmed on a server. For further details, all the different program flows with their corresponding explanations can be found in [33].

\section{CONClusions AND FUtURE WORK}

In this paper a simple but yet fully functional blockchain based P2P energy trading platform was presented and explained. All the code for developing the platform has been shared and the functionalities of the platform explained in detail. With this work the authors wanted to demonstrate how the whole trading framework can be implemented in a simple way using open source solutions. This work intends to be a stepping stone for researchers to investigate in this line. In future works we will add real advanced metering infrastructure with actual energy consumption/generation and more complex and automatize trading routines.

\section{APPENDIX: PlatForm CODE}

The whole platform code can be downloaded from: http://dx.doi.org/10.21227/hc2g-9807

\section{ACKNOWLEDGMENT}

The authors would like to express our gratitude to Adriano Mones from Phoenix Contact E-Mobility for believing in this project and for his constant interest and support.

\section{REFERENCES}

[1] International Energy Agency, "Renewables 2017," https://www.iea.org/, International Energy Agency (IEA), Tech. Rep., 2017.

[2] J. Conti, P. Holtberg, J. Diefenderfer, A. LaRose, J. T. Turnure, and L. Westfall, "International energy outlook 2016 with projections to 2040," USDOE Energy Information Administration (EIA), Washington, DC (United States), Tech. Rep., 2016.

[3] P. Siano, G. De Marco, A. Rolan, and V. Loia, "A Survey and Evaluation of the Potentials of Distributed Ledger Technology for Peer-to-Peer Transactive Energy Exchanges in Local Energy Markets," IEEE Syst. J., pp. 1-13, 2019.

[4] M. Pipattanasomporn, M. Kuzlu, and S. Rahman, "A Blockchain-based Platform for Exchange of Solar Energy: Laboratory-scale Implementation," Proc. Conf. Ind. Commer. Use Energy, ICUE, no. October, pp. 1-9, 2019.

[5] Z. Su, Y. Wang, Q. Xu, M. Fei, Y. C. Tian, and N. Zhang, "A Secure Charging Scheme for Electric Vehicles with Smart Communities in Energy Blockchain,” IEEE Internet Things J., 2018.

[6] A. S. Musleh, G. Yao, and S. M. Muyeen, "Blockchain applications in smart grid-review and frameworks," IEEE Access, vol. 7, pp 86 746-86757, 2019.

[7] K. Valtanen, J. Backman, and S. Yrjl, "Blockchain-Powered Value Creation in the 5G and Smart Grid Use Cases," IEEE Access, vol. 7, pp. $25690-25707,2019$.

[8] Z. Su, Y. Wang, Q. Xu, M. Fei, Y. Tian, and N. Zhang, "A Secure Charging Scheme for Electric Vehicles With Smart Communities in Energy Blockchain," IEEE Internet of Things Journal, vol. 6, no. 3, pp. 4601-4613, 2019.

[9] S. Wang, C. Huang, J. Li, Y. Yuan, and F. Wang, "Decentralized Construction of Knowledge Graphs for Deep Recommender Systems Based on Blockchain-Powered Smart Contracts," IEEE Access, 2019.

[10] H. Wang, Q. Wang, D. He, Q. Li, and Z. Liu, "BBARS: Blockchain-Based Anonymous Rewarding Scheme for V2G Networks," IEEE Internet of Things Journal, vol. 6, no. 2, pp. 3676-3687, 2019.

[11] Y. Wang, Z. Su, and N. Zhang, "BSIS: Blockchain-Based Secure Incentive Scheme for Energy Delivery in Vehicular Energy Network," IEEE Transactions on Industrial Informatics, vol. 15, no. 6, pp. 3620-3631, 2019.

[12] Z. Li, J. Kang, R. Yu, D. Ye, Q. Deng, and Y. Zhang, "Consortium Blockchain for Secure Energy Trading in Industrial Internet of Things," IEEE Transactions on Industrial Informatics, vol. 14, no. 8, pp 3690-3700, 2018.
[13] M. L. Di Silvestre, P. Gallo, M. G. Ippolito, R. Musca, E. Riva Sanseverino, Q. Thi Tu Tran, and G. Zizzo, "Ancillary Services in the Energy Blockchain for Microgrids," IEEE Transactions on Industry Applications, 2019.

[14] M. L. Di Silvestre, P. Gallo, M. G. Ippolito, E. R. Sanseverino, and G. Zizzo, "A Technical Approach to the Energy Blockchain in Microgrids," IEEE Transactions on Industrial Informatics, vol. 14, no. 11, pp. 4792-4803, nov 2018.

[15] J. Wang, Q. Wang, and N. Zhou, "A Decentralized Electricity Transaction Mode of Microgrid Based on Blockchain and Continuous Double Auction," in 2018 IEEE Power Energy Society General Meeting (PESGM), 2018, pp. 1-5.

[16] F. Luo, Z. Y. Dong, G. Liang, J. Murata, and Z. Xu, "A Distributed Electricity Trading System in Active Distribution Networks Based on Multi-Agent Coalition and Blockchain," IEEE Transactions on Power Systems, vol. 34, no. 5, pp. 4097-4108, 2019.

[17] A. Pinna, S. Ibba, G. Baralla, R. Tonelli, and M. Marchesi, "A Massive Analysis of Ethereum Smart Contracts Empirical Study and Code Metrics," IEEE Access, vol. 7, pp. 78 194-78213, 2019.

[18] C. Liu, K. K. Chai, X. Zhang, E. T. Lau, and Y. Chen, "Adaptive Blockchain-Based Electric Vehicle Participation Scheme in Smart Grid Platform," IEEE Access, vol. 6, pp. 25 657-25 665, 2018.

[19] J. Coignard, E. Munsing, J. MacDonald, and J. Mather, "Co-simulation Framework for Blockchain Based Market Designs and Grid Simulations," in 2018 IEEE Power Energy Society General Meeting (PESGM), 2018, pp. 1-5.

[20] S. Wang, A. F. Taha, and J. Wang, "Blockchain-Assisted Crowdsourced Energy Systems," in 2018 IEEE Power Energy Society General Meeting (PESGM), 2018, pp. 1-5.

[21] C. Sijie and L. Chen-Ching, "From Demand Response to Transactive Energy: State of the Art," Journal of Modern Power Systems and Clean Energy, vol. 5, no. 1, pp. 10-19, 2017.

[22] A. K. Bejestani, A. Annaswamy, and T. Samad, "A Hierarchical Transactive Control Architecture for Renewables Integration in Smart Grids: Analytical Modeling and Stability," IEEE Transactions on Smart Grid, vol. 5, no. 4, pp. 2054-2065, 2014.

[23] Z. Liu, Q. Wu, S. Huang, and H. Zhao, "Transactive Energy: A Review of State of the Art and Implementation," in 2017 IEEE Manchester PowerTech. IEEE, 2017, pp. 1-6.

[24] S. Kesting, F. Bliek, and F. Sioshansi, "From Consumer to Prosumer: Netherland's PowerMatching City Shows The Way," in Energy Efficiency. Academic Press, 2013, pp. 355-373.

[25] S. Kapferer, "Blockchain in the Energy Sector The Potential for Energy Providers," shorturl.at/qwLQY, May 2018.

[26] Verv, "Verv VLUX Whitepaper - The Evolution of Energy," https://verv.energy/, 2018.

[27] W. Gao, W. G. Hatcher, and W. Yu, "A Survey of Blockchain: Techniques, Applications, and Challenges," in 2018 27th International Conference on Computer Communication and Networks (ICCCN). IEEE, 2018, pp. 1-11

[28] F. Liang, W. Yu, D. An, Q. Yang, X. Fu, and W. Zhao, "A Survey on Big Data Market: Pricing, Trading and Protection," IEEE Access, vol. 6, pp. $15132-15154,2018$.

[29] F. B. Sean Ratka and A. Anisie, "IRENA Blockchain Innovation Landscape Brief,' International Renewable Energy Agency (IRENA), Tech. Rep., Feb. 2019.

[30] M. Andoni, V. Robu, D. Flynn, S. Abram, D. Geach, D. Jenkins, P. McCallum, and A. Peacock, "Blockchain Technology in the Energy sector: A Systematic Review of Challenges and Opportunities," Renew. Sustain. Energy Rev., vol. 100, no. October 2018, pp. 143-174, 2019.

[31] I. El-Sayed, P. Arboleya, K. Khan, and X. Dominguez, "A real pilot-platform implementation forblockchain-based peer-to-peer energy trading," 2019. [Online]. Available: http://dx.doi.org/10.21227/hc2g-9807

[32] Energy Web Foundation, "The Energy Web," https://www.energyweb.org/reports/the-energy-web-chain/, 2017.

[33] K. Khan, "Blockchain Based Peer-to-Peer Energy Trading using IoT Devices," Master's thesis, University of Oviedo, Spain, 2019. 